\title{
A Remote Design of MPC System with Model Diagnosis and Model Adjustment
}

\author{
Guoli $\mathrm{Ji}^{1, *}$, Xiaozhong $\mathrm{Li}^{1}$, Xiaorong $\mathrm{Hu}^{1}$ and Qianbin Huang ${ }^{1}$ \\ ${ }^{1}$ Department of Automation, Xiamen University, Xiamen, China \\ *Corresponding author: glji@xmu.edu.com
}

Keywords: Model predictive control; Model diagnosis; Model adjustment; Remote design

\begin{abstract}
This paper presents a remote design of model predictive control (MPC) system with model diagnosis and model adjustment. The design incorporates the model diagnosis and model adjustment into MPC. Furthermore, the MPC, model diagnosis and model adjustment are integrated in the remote controllable MPC system. The technologies of .NET, XML-RPC, Ajax asynchronous interaction and plugin are adopted. To enhance the MPC operating performance, the diagnosis module and adjustment module are designed as independent plugins of MPC. Implementations of the design will benefit the practical application of MPC as well as promoting the acceptability and usage of MPC in industry.
\end{abstract}

\section{Introduction}

In the last three decades, model predictive control (MPC) has been developed to meet the process industrial needs of power plants and petroleum refineries. So far it has already been applied in chemicals, food processing, aerospace and many other areas [1]. The DMC+(AspenTech) and Profit Controller (Honeywell) represent the fourth generation technology of MPC, which has new advantages such as high efficiency and accuracy of identification, robust control design and steady-state optimization analysis [1,2]. The Tai-Ji Control company has proposed the next-generation MPC technology, in which the commissioning and maintenance would be performed by the adaptive MPC automatically (Tai-Ji MPC System) [2].

The MPC system usually performs well at first. However, the controller performance may degrade, sometimes even break down over time because of the model mismatch which is caused by the constantly changing objects and environment. In the industrial process, the complexity of model maintenance and the cost of adjustment are keys to the successful operation of the control system [3]. In addition, the operating mode of MPC systems is still in local surveillance and requires the guidance of engineers. People from different fields, such as vendors, consumers and developers, have increasing requirements of the practicability of the MPC system.

This paper proposes a conceptual design of a remote MPC system with model diagnosis and model adjustment, which offers modules of online control, system identification, model diagnosis, model adjustment and remote monitoring. In the aspect of model diagnosis and adjustment, two modules are designed as two independent plugins of MPC. The plugins design may help the MPC system diagnosis the process model online, and adjustment of the model makes the MPC system more effective and reliable. Then we extend the MPC to be a long-distance system in order to help engineers observe the running status of the MPC on the remote as well as industry data and provide guidance. In the remote location MPC system would adjust the mismatch model automatically. With all these characteristics, the MPC may be more practical in industrial application.

\section{The design architecture of remote MPC system}

The design architecture consists of four modules (see Fig. 1): (1) the model predictive control module includes model identification and control; (2) model diagnosis module includes test signals addition and online model diagnosis; (3) model adjustment module includes mismatch model modification; (4) remote monitoring module includes data analysis and online control. 


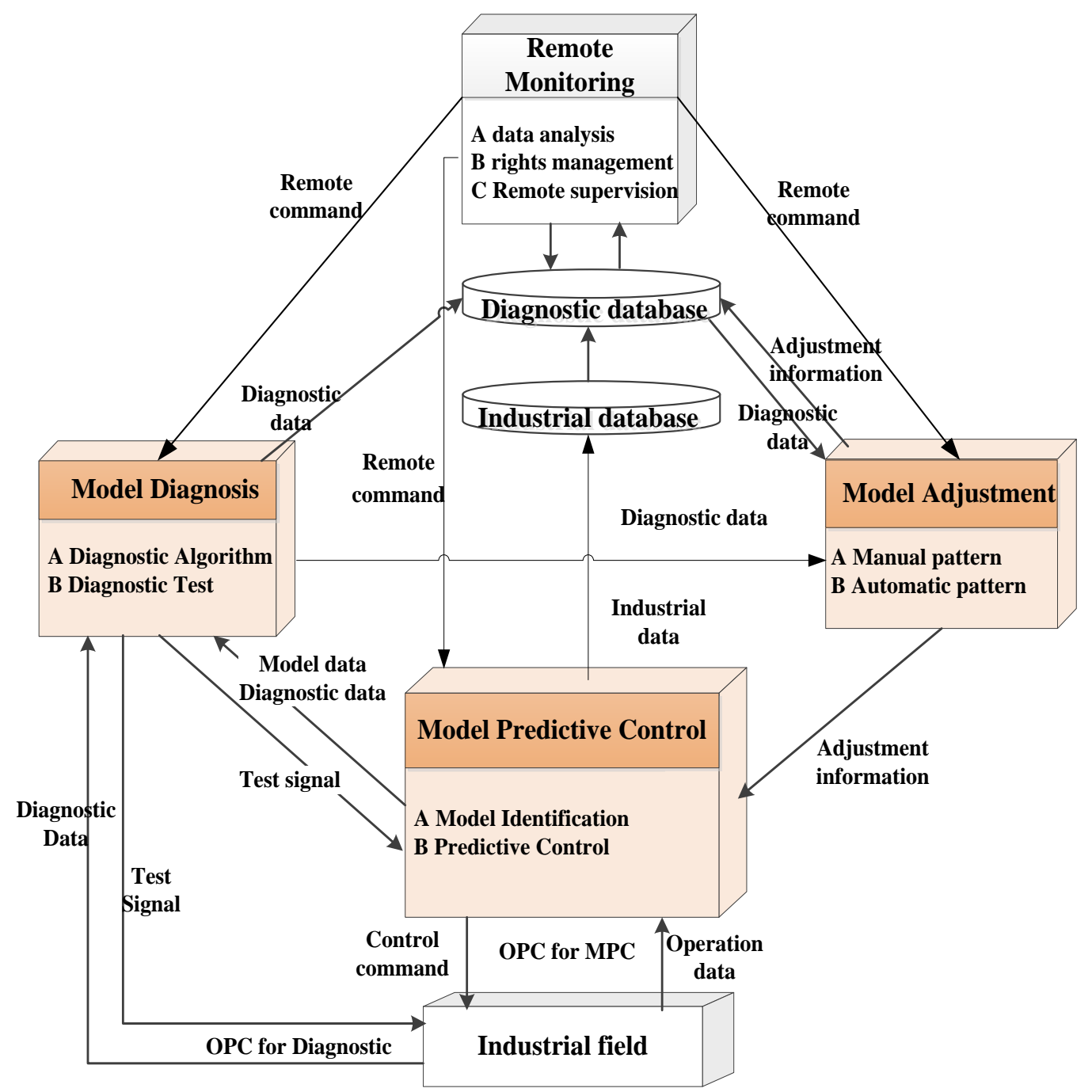

Fig. 1 Schematic Design of Remote MPC with Model Diagnosis and Adjustment

In the process of operation, the diagnosis, adjustment and monitoring are independent and coordinated plugins associated with MPC. In the entire system, model diagnosis and model adjustment are carried out under the closed-loop condition, to improve the practicability and reliability of MPC. Experts or engineers would solve the operation problem of MPC system in time on the remote end.

\subsection{MPC}

This paper adopts Tai-Ji MPC (Tai-Ji Control Inc.) [4], which consists of controller and model identification. During the MPC operation, the identification module performs automated plant test and automatic model identification. When all models are appropriate, the MPC controller is begins working. During each control sampling interval, the MPC control algorithm includes three steps: prediction, steady state optimization and dynamic control to accomplish the predict control [4].

\subsection{Model diagnosis}

In the early operation, MPC controller has a satisfactory performance under the supervision of experts and the model is good. But as the time goes on, the controllers may not achieve the expected control performance and even seriously deteriorate because of the model mismatch. To address this problem, a method of model error detection was proposed for MPC performance diagnosis [5]. Adding non-disturbing small sinusoidal test signals to diagnose the model and use plugin technology to combine model error detection into MPC system [6]. 


\subsection{Model adjustment}

Once the control performance becomes poor, model adjustment module will obtain the model error information through the diagnosis module. The model error index matrix ERR is defined as Eq.1:

$$
E R R_{r j}=\alpha_{1} \frac{\left|\hat{\hat{G}}_{N r j}\left(e^{\omega_{1} i}\right)-\hat{G}_{N r j}\left(e^{\omega_{1} i}\right)\right|}{\hat{\hat{G}}_{N r j}\left(e^{\omega_{1} i}\right)}+\alpha_{2} \frac{\left|\hat{\hat{G}}_{N r j}\left(e^{\omega_{2} i}\right)-\hat{G}_{N r j}\left(e^{\omega_{2} i}\right)\right|}{\hat{\hat{G}}_{N r j}\left(e^{\omega_{2} i}\right)}+\alpha_{3} \frac{\left|\hat{\hat{G}}_{N r j}\left(e^{\omega_{3} i}\right)-\hat{G}_{N r j}\left(e^{\omega_{3} i}\right)\right|}{\hat{\hat{G}}_{N r j}\left(e^{\omega_{3} i}\right)}
$$

where $\alpha_{1}+\alpha_{2}+\alpha_{3}=1, \hat{\hat{G}}_{N r j}\left(e^{\omega_{k} t}\right), \hat{G}_{N r j}\left(e^{\omega_{k} t}\right)$ are the r-output j-input frequency responses of the estimated model and the current MPC model respectively. The MPC user can monitor the model error index matrix, and compare it with some thresholds. When the model error exceeds the threshold, process data associated with re-identification command are sent to MPC controller by adjustment module. Thenceforwards, the replacement occurred for the sake of improving performance. Note that the threshold can be set differently according to given different MPC control requirement.

\subsection{Remote monitoring}

The design combines field monitoring and remote monitoring together, and the work environment can be divided into industrial field and Internet field [7]. The MPC software computers located around the industrial field are connected together by the internal LAN to form the MPC terminal. The MPC terminal stores data into the industrial database. The industrial local area network and remote end are linked according to the standardization of network communication. On the one hand, the data can be showed to the users in intuitive chart forms. On the other hand, MPC, model diagnosis and model adjustment can receive control command from the remote end. To ensure the consistency and security of the remote control, commands from the remote end will go through security filtering and command sorting before transmitting to the MPC terminals. In summary, remote monitoring can complete a series of operations containing the real-time data acquisition, identification test, diagnosis test and online control. It provides approach for the industrial site emergency treatment, and meets the requirements of online communication, data mining, and consulting services.

\section{Adopted techniques of remote MPC system}

The model diagnosis and model adjustment are integrated into MPC system based on plugin technology. The .NET three-tier architecture is used to design MPC remote end application. XML-RPC is selected to realize the communication between the local integrated MPC system and remote monitoring end. Ajax technology enhances the interactivity between the MPC and the remote end.

\subsection{Design pattern based on the.NET framework}

The remote MPC system design architecture is based on .NET three-tier architecture. Using hierarchical thought to make different layers of the remote end relatively independent and loosely coupled, and to build a clear architecture of system with high expansibility and maintainability. The .net three-tier architecture includes presentation layer, business logic layer, and data access layer. Reasonable organization of layers makes sure the reliable operation of the remote end.

\subsection{Interaction mechanisms of the design}

This section discusses the dynamic relationships among the components mentioned above. In the local MPC system, plugin technology is adopted to integrate model diagnosis and model adjustment into MPC system; while in remote end, XML-MPC and Ajax technology are adopted to combine MPC with remote end.

\subsubsection{Interactive architecture based on plugin technology}

Plugin architecture is a kind of flexible technology. We set the model diagnosis and model adjustment as separated plugins which can be developed independently. Each plugin module can be 
inserted into the MPC system dynamically and is free to remove or replace, to maintain the origninal functions unchanged.

\subsubsection{Communication mechanisms based on XML-RPC}

XML-RPC distributed technology is adopted to achieve the long-distance realization.. In operation, users first send service call request to web, then the web turns the request into XML form. As per the HTTP, send the request information to the remote client terminal [8]. Through analyzing the XML-RPC information, the remote client receives command to finish relevant operation. In a similar way, the remote client would send information to service requester.

\subsubsection{Interactive technology based on Ajax}

To guarantee the timely communication between the remote end and the local MPC, Ajax technology is adopted because it can make an asynchronous call for the page refresh [9]. Using an asynchronous call does not need to interrupt the user action, on the contrary, it realizes the remote data interaction and update pages without refreshing. Ajax technology has obvious advantages: reducing redundancy client requests, shortening the response time of service and providing users a more personalized and convenient service.

\section{Conclusion and Perspective}

In conclusion, we propose a remote design of a MPC system with model diagnosis and model adjustment, which improves the practicability and reliability of MPC. Introducing the remote MPC system into operation will promote the acceptance as well as the widespread applications. With the development of the Internet technology and the deep research of MPC theory, the realization of remote MPC system becomes possible in the future. Future directions will focus on (1) adding more diagnosis algorithms to meet different requirements; (2) improving model adjustment method to realize the closed-loop automatic adjustment of mismatch model.

\section{Acknowledgements}

This work was supported by the National Natural Science Foundation of China [61174161, 61304141, 61375077]; the specialized Research Fund for the Doctoral Program of Higher Education of China [20130121130004]; and the Fundamental Research Funds for the Central Universities in China (Xiamen University: No. CBX2014038).

\section{References}

[1] S. Joe.Qin, Thomas A. Badgwell: Control Engineering Practice Vol.11 (2003) p. 733-764.

[2] Y.C.Zhu, R. Patwardhan, S.B.Wagner and J. Zhao: Computers and Chemical EngineeringVol.51 (2013) p.124-135

[3] M.Morari, J.H.Lee:Computers and Chemical Engineering Vol.23 (1999) p.667-682

[4] Information on http://www.taijicontrol.com

[5] G.L.Ji, K.K.Zhang, Y.C.Zhu :Journal of Process Control, Vol. 22.3(2012) p. 635-642.

[6] G.L.Ji, L.L.Chen, Y.Luo, J.C Lin, et al: Engineering (NUiCONE), 2013 p. 1-5

[7] G.L.Ji, X.L.Liu, K.K.Zhang, W.Lin, Q.Wang,et.al: Innovations in Intelligent Systems and Applications(2011) p.19-23

[8] G.G.de Rivera, R.Ribalda, J.Colas and J.Garrido: Advanced Intelligent Mechatronics(2005) p. 1336-1341

[9] L.D.Paulson: Computer Vol. 38.10(2005) p. 14-17. 